\title{
The Model-Matching Error and Optimal Solution in Locally Convex Space
}

\author{
Lixin Ma \\ Department of Mathematics, Dezhou University, Dezhou 253023, China \\ E-mail: malixin6019@yahoo.com.cn
}

\begin{abstract}
The model-matching error and the optimal solution in the Hardy space are extended to the locally convex space, and the model-matching error and the optimal solution in the locally convex space are achieved. Thereby the ordinary $H_{\infty}$-control theory is extended to with range in locally convex spaces through a form of a parameter vector. The algorithms of computing the infimal model-matching error and the infimal controller are presented.
\end{abstract}

Keywords: Locally convex space, Inner-outer function, Minimal realization, Infinal model- matching error

\section{INTRODUCTION}

Assume that $R$ is the real field and $R^{n}$ is the Cartesian product of $n$ copies of $R$, here $n$ is any positive integer, and that $C$ is a complex plane.

To solve the problem for simplicity, we apply the $G(s)$ in the model matching problem to $G(s, \xi)$, where $s$ in $C, \xi$ in $R^{n}$, and $G(s, \xi)$ is in $C^{\infty}\left(R^{n}\right)$ (locally convex space) for each fixed $s$ in $C$ and in $H_{\infty}$ for each fixed $\xi$ in $R^{n}$. First, we extend several concepts.

Definition 1 The locally convex space $V H_{\infty}$ consists of all complex-valued parameter functions $F(s, \xi)$ of a complex variable $s$ and a parameter $\xi$ which are analytic and bounded about $s$ in $\operatorname{Re} s>0$ (for each fixed $\xi$ in $\left.R^{n}\right)$. Similarly, we define the $V H_{\infty}$-norm of $F(s, \xi)$ is

$$
\|F\|_{\infty}=\sum_{k=1}^{\infty} \frac{g_{k}}{2^{k}\left(1+g_{k}\right)},
$$

where $g_{k}=\sup _{-k<\xi<k}\|F(\bullet, \xi)\|_{\infty}$

Definition 2 The subset of $V H_{\infty}$ consists of all real-rational functions of $s$ and $\xi$,will be denoted by $V R H_{\infty}$.

Definition 3 Let $\alpha$ denote the infimal model-matching error

$$
\alpha=\inf \left\{\left\|\mid T_{1}-T_{2} Q T_{3}\right\|_{\infty}: Q \in V R H_{\infty}\right\} .
$$

A matrix $Q$ in $V R H_{\infty}$ satisfying $\alpha=\left\|T_{1}-T_{2} Q T_{3}\right\|_{\infty}$ will be called optimal,where $\alpha$ is a model-matching error. When $T_{i}(s, \xi)$ are scalar-valued,then there is no need for both $T_{2}(s, \xi)$ and $T_{3}(s, \xi)$. So we may as well suppose $T_{3}(s, \xi)=1$.It is also assumed that $T_{2}^{-1}(s, \xi) \in V R H_{\infty}$ to avoid the trivial instance of the problem.

Returning to the model-matching problem, bring in an inner-outer factorization of $T_{2}(s, \xi): T_{2}(s, \xi)=T_{2 i}(s, \xi) T_{2 o}(s, \xi)$,

we have

$$
\left\|T_{1}-T_{2} Q\right\|_{\infty}=\|R-X\|_{\infty} .
$$


We conclude that

$$
\alpha=\inf \left\{|| R-X \mid \|_{\infty}: X \in V R H_{\infty}\right\}=\operatorname{dist}\left(R, V R H_{\infty}\right) .
$$

Definition 4 The $V L_{p}$ space, $1 \leq p<\infty$, will be viewed as $p$ th power integrable functions about $S$ and $\xi$.When $p=\infty, V L_{\infty}$ is the space of essentially bounded functions(for any fixed $\xi$ in $R^{n}$ ).

Definition 5 The $V R L_{p}$ space, $V R L_{p}$, will be viewed as a subset of $V L_{p}$, which consists of all real-rational functions of $S$ and $\xi$.

Definition 6

(i) Let $F(s, \xi) \in V L_{\infty}$ and $g(s, \xi) \in V L_{2}$.Then the operator

$$
\Lambda_{F(s, \xi)}: \Lambda_{F(s, \xi)} g(s, \xi)=F(s, \xi) g(s, \xi)
$$

is called the Laurent operator.

(ii) A related operator is $\Lambda_{F(s, \xi)} \mid V H_{2}$, the restriction of

$\Lambda_{F(s, \xi)}$ to $V H_{2}$, which maps $V H_{2}$ to $V L_{2}$, where $F(s, \xi) \in V L_{\infty}$.

(iii) For $\mathrm{t} F(s, \xi) \in V L_{\infty}$, the Hankel operator with symbol $F(s, \xi)$, denoted by $\Gamma_{F(s, \xi)}$, maps $V H_{2}$ to $V H_{2}{ }^{\perp}$ and is defined as

$$
\Gamma_{F(s, \xi)}=\Pi_{1} \Lambda_{F(s, \xi)} \mid V H_{2},
$$

where $V L_{2}=V H_{2} \oplus V H_{2}{ }^{\perp}$, and $\Pi_{1}$ is the projection from $V L_{2}$ onto $V H_{2}{ }^{\perp}$.

Definition 7 We call $F(s, \xi)$ to be strong proper if $F(s, \xi) \in V R H_{\infty}$ and $\sup _{\xi \in R^{n}} \mid F(\bullet, \xi \mid<\infty$, strictly strong proper if $F(\infty, \xi) \equiv 0$.

Definition 8 We call $F(s, \xi)$ to be stable if $F(s, \xi) \in V R H_{\infty}$ and $F(s, \xi)$ has no poles in the closed right half-plane $\operatorname{Re} s \geq 0$ ( for each fixed $\xi$ in $R^{n}$ ).

If $F(s, \xi)$ is real-rational about $s$ in $\operatorname{Re} s>0$, then $F(s, \xi) \in V R H_{\infty}$ if and only if $F$ is strong proper and stable ( for each fixed $\xi$ in $R^{n}$ ). Similarly, we define

$$
G(s, \xi)=\left[\begin{array}{ll}
T_{1}(s, \xi) & T_{2}(s, \xi) \\
T_{2}(s, \xi & 0
\end{array}\right], K(s, \xi)=-Q(s, \xi),
$$

then the model-matching problem is

$$
\text { || } T_{1}-T_{2} Q T_{3} \|=\min \text { imum, }
$$

where $T_{i}(i=1,2,3) \in V R H_{\infty}$. The constraint that $K$ stabilizes $G$ is equivalent to that $Q \in V R H_{\infty}$.

We shall give in the form of parameter valued case the algorithms of computing the model-matching error $\alpha$ and the optimal controller $Q$.

\section{THE MINIMAL REALIZATION}

Definition 9 The linear time invarient system $S_{1}$ defined by

$$
\begin{gathered}
x(t, \xi)=A(\xi) x(t, \xi)+B(\xi) u(t, \xi) \\
y(t, \xi)=C(\xi) x(t, \xi)
\end{gathered}
$$

Where $A(\xi)$ is $n \times n, B(\xi)$ is $n \times m$, and $C(\xi)$ is $r \times n$ constant matrix depending on $\xi$, is said to be completely controllable if the $n \times m n$ controllability matrix

$$
U(\xi)=\left[B(\xi), A(\xi) B(\xi), \ldots, A^{n-1}(\xi) B(\xi)\right]
$$

has rank $n$,denoted by $(A(\xi), B(\xi))$.

Definition 10 The system $S_{1}$ described by (1) and (2) is completely observable if the observability matrix 


$$
V^{T}(\xi)=\left[C(\xi), C(\xi) A(\xi), \ldots, C(\xi) A^{n-1}(\xi)\right]^{T}
$$

has rank $n$,denoted by $(A(\xi), C(\xi))$.

Definition 11 Given an $r \times m$ matrix $G(s, \xi)$ whose elements are rational functions of $s$, we wish to find matrices $A(\xi), B(\xi)$ and $C(\xi)$ depending on $\xi$,having dimensions $n \times n, n \times m$ and $r \times n$ respectively, such that

$$
G(s, \xi)=C(\xi)\left(s I_{n}-A(\xi)\right)^{-1} B(\xi)(5)
$$

where $I_{n}$ is the unit matrix of order $n$.

$[A(\xi), B(\xi), C(\xi), 0]$ is termed a realization of $G(s, \xi)$ of order $n$, and is not, of course, unique. All such the above realizations will include matrices $G(s, \xi)$ having the least dimensions-be called the minimal realizations.

Definition 12 The Laplace transform of parameter-valued function $f(s, \xi)$ is defined by

$$
F(s, \xi)=\int_{0}^{\infty} f(t, \xi) e^{-s t} d t=L f(t, \xi)
$$

and the inverse Laplace transform of $F(s, \xi)$ is

$$
f(t, \xi)=\int_{\sigma-j \infty}^{\sigma+j \infty} F(s, \xi) e^{s t} d s=L^{-1} F(s, \xi)
$$

we take the Laplace transform of (9) with zero initial conditions, we have

$$
\hat{s x}(s, \xi)=A(\xi) \hat{x}(s, \xi)+B(\xi) \hat{u}(s, \xi)
$$

and after rearrangement

$$
\hat{x}(s, \xi)=\left(s I_{n}-A(\xi)\right)^{-1} B(\xi) \hat{u}(s, \xi)
$$

Since from (10) the Laplace transform of the output is

$$
\hat{y}(s, \xi)=C(\xi) \hat{x}(s, \xi)
$$

clearly

$$
\hat{y}(s, \xi)=C(\xi)\left(s I_{n}-A(\xi)\right)^{-1} B(\xi) \hat{u}(s, \xi)=G(s, \xi) \hat{u}(s, \xi)
$$

where the $r \times m$ matrix

$$
G(s, \xi)=C(\xi)\left(s I_{n}-A(\xi)\right)^{-1} B(\xi)
$$

Suppose $R(S, \xi)=\left[r_{i j}(s, \xi)\right]$ is an $p \times m$ strictly proper rationalfraction matrix of $S$ (for any fixed $\xi$ in $R^{n}$ ).

Theorem 1 A realization $[A(\xi), B(\xi), C(\xi), 0]$ of a given transfer matrix $G(s, \xi)$ is minimal if $(A(\xi), B(\xi))$ is c.c. and $(A(\xi), C(\xi)) \quad$ c.o.

Proof Let $U(\xi)$ and $V(\xi)$ be the controllability and observability matrices in (5) and (6) respectively. We wish to show that if these both have rank $n$ then $R(s, \xi)$ has

least order $n$.

Suppose that there exists a realization $\{\bar{A}(\xi), \bar{B}(\xi), \bar{C}(\xi)\}$

of $R(s, \xi)$, with $\bar{A}(\xi)$ having order $n_{1}$. Since

$$
C(\xi)\left(s I_{m}-A(\xi)\right)^{-1} B(\xi)=\bar{C}(\xi)\left(s I_{m}-\bar{A}(\xi)\right)^{-1} \bar{B}(\xi),
$$

It follows that

$$
C(\xi) e^{A(\xi) t} B(\xi)=\bar{C}(\xi) e^{\bar{A}(\xi) t} \bar{B}(\xi)
$$

Which implies, using the series

$\left(e^{A(\xi) t}=I+t A(\xi)+\frac{t^{2}}{2} A^{2}(\xi)+\ldots\right)$, that

$$
C(\xi) A^{i}(\xi) B(\xi)=\bar{C}(\xi) \bar{A}^{i}(\xi) \bar{B}(\xi) \quad i=0,1,2, \ldots
$$


Consider the product

$$
\begin{aligned}
V(\xi) U(\xi) & =\left[\begin{array}{l}
C(\xi) \\
C(\xi) A(\xi) \\
\cdot \\
C(\xi) A^{n-1}(\xi)
\end{array}\right]\left[B(\xi), A(\xi) B(\xi), \ldots, A^{n-1}(\xi) B(\xi)\right] \\
& =\left[\begin{array}{ll}
C(\xi) B(\xi) & C(\xi) A^{n-1}(\xi) B(\xi) \\
\cdot & \cdot \\
C(\xi) A^{n-1}(\xi) & C(\xi) A^{2 n-2}(\xi) B(\xi)
\end{array}\right] \\
& =\left[\bar{B}(\xi), \bar{A}(\xi) \bar{B}(\xi), \ldots, \bar{A}^{n-1}(\xi) \bar{B}(\xi)\right]=V_{1}(\xi) U_{1}(\xi) .
\end{aligned}
$$

By assumping, $V(\xi)$ and $U(\xi)$ both have rank $n$, so the matrix $V_{1}(\xi) U_{1}(\xi)$ also have rank $n$. However, the dimension of $V_{1}(\xi)$ and $U_{1}(\xi)$ are respectively $r_{1} n \times n_{1}$ and $n_{1} \times m_{1} n$, where $r_{1}$ and $m_{1}$ are positive integers, so that the rank of ix $V_{1}(\xi) U_{1}(\xi)$ can not be greater than $n_{1}$. That is, $n<n_{1}$, so there can be no realization of $G(s, \xi)$ having order less than $n$.

\section{INFIMAL MODEL-MATCHING ERROR}

The Lyapunov equations are

$$
\begin{aligned}
& A(\xi) L_{c}(\xi)+L_{o}(\xi) A^{\mathrm{T}}(\xi)=B(\xi) B^{\mathrm{T}}(\xi) \\
& A^{\mathrm{T}}(\xi) L_{o}(\xi)+L_{o}(\xi) A(\xi)=C^{\mathrm{T}}(\xi) C(\xi)
\end{aligned}
$$

Define the two controllability and observability gramians:

$$
\begin{aligned}
& L_{c}(\xi)=\int_{0}^{\infty} e^{-A(\xi) t} B(\xi) B^{\mathrm{T}}(\xi) e^{-A^{\mathrm{T}}(\xi) t} d t, \\
& L_{o}(\xi)=\int_{0}^{\infty} e^{-A^{\mathrm{T}}(\xi) t} C^{\mathrm{T}}(\xi) C(\xi) e^{-A(\xi) t} d t .
\end{aligned}
$$

Theorem $2 L_{c}(\xi)$ and $L_{o}(\xi)$ are the unique solutions of (12) and (13) respectively.

Proof Using the definition we have $A(\xi) L_{c}(\xi)+L_{c}(\xi) A^{\mathrm{T}}(\xi)$

$$
\begin{aligned}
= & \int_{0}^{\infty}\left(A(\xi) e^{-A(\xi) t} B(\xi) B^{\mathrm{T}}(\xi) e^{-A^{\mathrm{T}}(\xi) t}+=B(\xi) B^{\mathrm{T}}(\xi)-\lim _{t \leftarrow \infty}\left(e^{-A(\xi) t} B(\xi) B^{\mathrm{T}}(\xi) e^{-A^{\mathrm{T}}(\xi) t}\right) .\right. \\
\left.e^{-A(\xi) t} B(\xi) B^{\mathrm{T}}(\xi) e^{-A^{\mathrm{T}}(\xi)} A^{\mathrm{T}}(\xi)\right) d t &
\end{aligned}
$$

Since $A(\xi)$ is instable,

$$
\lim _{t \rightarrow \infty}\left(e^{-A(\xi) t} B(\xi) B^{\mathrm{T}}(\xi) e^{-A^{\mathrm{T}}(\xi) t}\right)=0 .
$$

So $L_{c}(\xi)$ are the unique solutions of (12).From the discussion above, the uniqueness is obvious.

$L_{o}(\xi)$ are the unique solutions of (13) follows similarly.

Definition 13 Suppose the linear operator

$$
T: X \rightarrow Y,
$$

it's the unique operator

$$
T^{*}: Y^{*} \rightarrow X^{*},
$$

Satisfying

$$
\left(T^{*} y^{*}, x\right)=\left(y^{*}, T x\right), x \in X^{*}, y \in T^{*},
$$

$T^{*}$ is called the adjoint of $T$.

Define 


$$
\begin{aligned}
& f(s, \xi)=[A(\xi), \omega(\xi), C(\xi), 0], \\
& g(s, \xi)=\left[-A^{\mathrm{T}}(\xi), \lambda^{-1}(\xi) L_{0}(\xi) \omega(\xi), B^{\mathrm{T}}(\xi), 0\right],
\end{aligned}
$$

and

$$
X(s, \xi)=R(s, \xi)-\lambda(\xi) f(s, \xi) / g(s, \xi) .
$$

So

$$
f(s, \xi)=C(\xi)(s I-A(\xi))^{-1} \omega(\xi) \in V R H_{2}{ }^{\perp},
$$

and

$g(s, \xi)=B^{\mathrm{T}}(\xi)\left(s I+A^{\mathrm{T}}(\xi)\right)^{-1} \lambda^{-1}(\xi) L_{o}(\xi) \omega(\xi) \in V R H_{2}$.

Theorem $3^{[4]}$ There exists a closest $V R H_{\infty}$-function $X(s, \xi)$ to a given $V R L_{\infty}$-function $R(s, \xi)$, and $\|R-X\|=\left\|\Gamma_{R}\right\|$.

Factor $R(s, \xi)$ as

$$
R(s, \xi)=R_{1}(s, \xi)+R_{2}(s, \xi)
$$

With $R_{2}(s, \xi)$ strictly proper and analytic in $\operatorname{Re} s<0$ and $R_{2}(s, \xi)$ in $V R H_{\infty}$. Then $R_{1}(s, \xi)$ has a minimal state-space realization

$$
R_{1}(s, \xi)=[A(\xi), B(\xi), C(\xi), 0]
$$

Define

$$
\begin{array}{r}
L_{c}(\xi)=\lambda(\xi) \omega(\xi) \\
L_{0}(\xi)=\lambda(\xi) v(\xi)
\end{array}
$$

Lemma 4 The function $f(s, \xi)$ and $g(s, \xi)$ satisfying equations

$$
\begin{aligned}
\Gamma_{R(s, \xi)} g(s, \xi) & =\lambda(\xi) f(s, \xi) \\
\Gamma^{*} R(s, \xi) & f(s, \xi)=\lambda(\xi) g(s, \xi)
\end{aligned}
$$

Proof to prove (21) start with (15). Add and subtract $s L_{c}(\xi)$ on the left-hand side to get

$$
-(s I-A(\xi)) L_{c}(\xi)+L_{c}(\xi)\left(s I+A^{\mathrm{T}}(\xi)\right)=B^{\mathrm{T}}(\xi) B(\xi)
$$

Now pre-multiply by $C(\xi)(s I-A(\xi))^{-1}$ and pre--multiply by $\left(s I+A^{\mathrm{T}}(\xi)\right)^{-1} v(\xi)$ to get

$$
\begin{aligned}
& -C(\xi) L_{c}(\xi)\left(s I+A^{\mathrm{T}}(\xi)\right) v(\xi)+C(\xi)(s I-A(\xi))^{-1} L_{c}(\xi) v(\xi) \\
& =C(\xi)(s I-A(\xi))^{-1} B(\xi) B^{\mathrm{T}}(\xi)\left(s I+A^{\mathrm{T}}(\xi)\right)^{-1} v(\xi)
\end{aligned}
$$

The first function on the left-hand side belong to $\mathrm{VH}_{2}$; from (17) and (19) the second function equals $\lambda(\xi) f(s, \xi)$; and from (18) and (19) the function on the right-hand side equals $R_{1}(s, \xi) g(s, \xi)$. Project both side of (23) onto $\mathrm{VRH}_{2}{ }^{\mathrm{T}}$ to get

$$
\lambda(\xi) f(s, \xi)=\Pi_{1} R_{1}(s, \xi) g(s, \xi)=\Gamma_{R_{1}(s, \xi)} g(s, \xi) .
$$

But $\Gamma_{R(s, \xi)}=\Gamma_{R_{1}(s, \xi)}$; hence (21) dolds.

Equation (22) is proved similarly starting with (16).

Q.E.D.

From Lemma 4, we can conceive

Corollary $5\left\|\Gamma_{R(s, \xi) \|}\right\|=\lambda(\xi)$

Theorem 6 The infimum model-matching error $\alpha$ equals $\lambda(\xi)$, the unique optimal $X$ equals

$$
R(s, \xi)-\lambda(\xi) \frac{f(s, \xi)}{g(s, \xi)} .
$$

Proof from Theorem 3 there exists a function $X(s, \xi)$ in $V H_{\infty}$ such that 


$$
\|R-X\|_{\infty}=\left\|\Gamma_{R(s, \xi)}\right\|
$$

It is claimed that every $X(s, \xi)$ in $V H_{\infty}$ satisfying (24) also satisfies

$$
R(s, \xi)-X(s, \xi) g(s, \xi)=\Gamma_{R(s, \xi)} g(s, \xi)
$$

But (25) has a unique solution, namely,

$$
X(s, \xi)=R(s, \xi)-\lambda(\xi) \frac{f(s, \xi)}{g(s, \xi)}
$$

Thus (21) and Theorem 3 imply

$$
\alpha(\xi)=\lambda(\xi)
$$

Therefore

$$
X(s, \xi)=R(s, \xi)-\alpha(\xi) \frac{f(s, \xi)}{g(s, \xi)}
$$

Set

$$
\alpha(\xi)=\lambda(\xi), \quad Q(s, \xi)=T_{2}^{-1}(s, \xi) X(s, \xi) .
$$

Since $T_{20}(s, \xi), T_{20}{ }^{-1}(s, \xi) \in V R H_{\infty},(26)$ sets up a one-to-one correspondence between functions $Q(s, \xi)$ in $V R H_{\infty}$ and functions $X(s, \xi)$ in $V R H_{\infty}$. An optimal $X(s, \xi)$ yields an optimal $Q(s, \xi)$ via (24)

For a single-input and single-output design in the form of parameter valued case, we have similar to ordinary computing method.

Example.

$$
\begin{aligned}
& P(s, \xi)=\frac{(s-1)(s-2)}{(s+1)\left(s^{2}+s+1+\xi^{2}\right)} \in V R H_{\infty}, \omega_{1}=0.01, \\
& \varepsilon=0.1 .
\end{aligned}
$$

From the above method, we derive

$$
K(s, \xi)=\frac{0.615(s+0.4)(s+1)\left(s^{2}+s+1+\xi^{2}\right)}{s^{4}+6.145 s^{3}+12.54 s^{2}+13.53 s+0.0232} .
$$

Note $K(s, \xi) \notin R H_{\infty}$, but $K(s, \xi) \in V R H_{\infty}$.

Step 1.

$$
\begin{aligned}
-P(s, \xi) & =\frac{N(s, \xi)}{M(s, \xi)}, \\
N(s, \xi) & =-P(s, \xi), M(s, \xi)=1=X(s, \xi), Y(s, \xi)=0 .
\end{aligned}
$$

Step 2.

$$
W(s, \xi)=\frac{s+1}{10 s+1} .
$$

Step 3.

$$
\begin{gathered}
T_{1}(s, \xi)=\frac{(s+1)^{k}}{(10 s+1)^{k}} \\
T_{2}(s, \xi)=-\frac{(s+1)^{k}(s-1)(s-2)}{(10 s+1)^{k}(s+1)\left(s^{2}+s+1+\xi^{2}\right)}, \\
V(s)=s+1 .
\end{gathered}
$$

Step 4. When $k=1$,

Step (1)

$$
\begin{aligned}
& T_{21}(s, \xi)=\frac{(s-1)(s-2)}{(s+1)(s+2)}, \\
& T_{20}=-\frac{(s+1)(s+2)}{(10 s+1)\left(s^{2}+s+1+\xi^{2}\right)} .
\end{aligned}
$$


Step (2)

$$
R(s, \xi)=\frac{(s+1)^{2}(s+2)}{(10 s+1)\left(s^{2}+s+1+\xi^{2}\right)}
$$

the minimal realization is

Step (3)

$$
A=\left[\begin{array}{ll}
1 & 0 \\
0 & 2
\end{array}\right], B=\left[\begin{array}{l}
-\frac{12}{11} \\
\frac{12}{7}
\end{array}\right], \quad C=\left[\begin{array}{ll}
1 & 1
\end{array}\right] .
$$

Step (4)

$$
L_{c}=\left[\begin{array}{cc}
\frac{72}{121} & -\frac{48}{77} \\
-\frac{48}{77} & \frac{36}{49}
\end{array}\right], \quad L_{0}=\left[\begin{array}{cc}
\frac{1}{2} & \frac{1}{3} \\
\frac{1}{3} & \frac{1}{4}
\end{array}\right] \text {. }
$$

$$
L_{c} L_{0}=\left[\begin{array}{ll}
0.0898 & 0.0425 \\
-0.0668 & -0.0853
\end{array}\right] \text {. }
$$

Then

$$
\alpha_{1}=0.2299>0.1 \text {. }
$$

When $k=2$,

Step (1)

$$
T_{21}(s, \xi)=\frac{(s-1)(s-2)}{(s+1)(s+2)},
$$

$$
T_{20}=-\frac{(s+1)(s+2)}{(10 s+1)\left(s^{2}+s+1+\xi^{2}\right)} .
$$

Step (2)

$$
R(s, \xi)=\frac{(s+1)^{3}(s+2)}{(10 s+1)^{2}\left(s^{2}+s+1+\xi^{2}\right)}
$$

the minimal realization is

Step (3)

$$
A=\left[\begin{array}{ll}
1 & 0 \\
0 & 2
\end{array}\right], \quad B=\left[\begin{array}{l}
-\frac{24}{121} \\
\frac{12}{49}
\end{array}\right], \quad C=\left[\begin{array}{ll}
1 & 1
\end{array}\right] .
$$

$$
L_{0}=\left[\begin{array}{ll}
\frac{1}{2} & \frac{1}{3} \\
\frac{1}{3} & \frac{1}{4}
\end{array}\right] .
$$

Step (4)

$$
\begin{aligned}
L_{c} & =\left[\begin{array}{cc}
\frac{24.12}{121.121} & -\frac{8.12}{121.49} \\
-\frac{8.12}{121.49} & \frac{12.3}{49.49}
\end{array}\right], \\
L_{c} L_{0} & =\left[\begin{array}{ll}
0.0044 & -0.0025 \\
0.0031 & -0.0017
\end{array}\right] .
\end{aligned}
$$

Then

$$
\alpha_{1}=0.05113<0.1, \quad \omega=\left[\begin{array}{l}
1 \\
-0.7209
\end{array}\right] .
$$

Step (5)

$$
\begin{gathered}
f(s,)=\frac{0.2791 s-1.2791}{(s-1)(s-2)} \\
g(s)=\lambda^{-1} \frac{-0.0141 s-0.0657}{(s+1)(s+2)} \\
X(s)=6.15 \frac{(s+1)(s+2)(s+0.4)}{(10 s+1)^{2}(s+4.66)} .
\end{gathered}
$$

Step(6) Set 


$$
\begin{gathered}
\alpha=\lambda=0.05113 \\
Q(s, \xi)=-6.15 \frac{(s+0.4)\left(s^{2}+s+1+\xi^{2}\right)}{(s+1)(s+4.66)} \\
Q_{\alpha}(s, \xi)=-6.15 \frac{(s+0.4)\left(s^{2}+s+1+\xi^{2}\right)}{(10 s+1)(s+1)(s+4.66)}, \\
K(s, \xi)=0.615 \frac{(s+0.4)(s+1)\left(s^{2}+s+1+\xi^{2}\right)}{s^{4}+6.145 s^{3}+12.54 s^{2}+13.53 s+0.0232} .
\end{gathered}
$$

Step 5 .

\section{References}

Francis, B.A. (1987). A course in $H_{\infty}$-Control Theory. Spring-Verlag, Berlin. Heidelberg. New York, 1987. 61-80.

Francis, B.A., \& Doyle, J.C. (1987). Linear Control Theory with an $H_{\infty}$-Optimality Criterion. SIAM control and Optimization, 1987. 25: 815-844.

Francis, B.A., \& Zames, G. (1984). On $H_{\infty}$-Optimal Sensitivity Theory for SISO Feedback System. IEEE Trans. Auto Cont, 1984. AC-29: 9-16.

Kerulen, B.V. (1993). $H_{\infty}$-Control with Measurement-Feedback for Infimite-Dimensional Systems. Journal Mathematical Systems, Estimation and control, 1993.3: 373-411. 